\title{
Susceptibility of rabbits to Treponema pallidum after infection with Mycobacterium bovis
}

\section{S. GRAVES}

From the Department of Microbiology, Monash University Medical School, Alfred Hospital, Prahran, Australia

SUMMARY Rabbits stimulated with Mycobacterium bovis (strain BCG) one month before challenge with Treponema pallidum (Nichols) did not show any modification in their development of syphilitic lesions. A second infection with BCG given at the same time and at the same intradermal site as the $T$. pallidum challenge also failed to protect the rabbits against syphilis. Thus the non-specific activation of cell-mediated immunity by BCG does not appear to protect rabbits against $T$. pallidum infection even when both activation and challenge take place in the dermis. The role of the macrophage in syphilis remains obscure.

\section{Introduction}

Human syphilis and the animal model disease (Treponema pallidum infection in rabbits) results in both a humoral and a cell-mediated immune response by the host (Kiraly, 1976; Musher et al., 1976), but the relative contribution of each to overall immunity has yet to be determined. Antibody alone is not fully protective, since in passive transfer experiments rabbits ultimately succumb to infection (Perine et al., 1973; Sepetjian et al., 1973; Turner et al., 1973; Graves and Johnson, 1975; Bishop and Miller, 1976; Weiser et al., 1976). This suggests that cell-mediated immune responses also play a part in the establishment of the immune state. There is evidence both for and against this (Levene et al., 1971; Metzger and Smogor, 1975; Schell and Musher, 1975; Schell et al., 1975a, b; Wicker and Wicker, 1975; Baughn et al., 1977b; Metzger et al., 1977; Wicker and Wicker, 1977).

Although activation of host macrophages is one of the main effector mechanisms of cell-mediated immunity, resulting in enhanced intra-macrophage killing of phagocytosed micro-organisms, it has been shown that the non-specific activation of rabbit macrophages by living Mycobacterium bovis (strain BCG) or Propionibacterium acnes did not render rabbits more resistant to a $T$. pallidum challenge

Address for reprints: Dr S. Graves, Department of Microbiology, Monash University Medical School, Alfred Hospital, Prahran, Victoria 3181, Australia

Received for publication 21 February 1979
(Graves and Johnson, 1975; Schell et al., 1975b; Baughn et al., 1977a).

The experiment reported here was devised to stimulate host immunity with BCG at the same site as the $T$. pallidum infection, the dermis. It was thought that the activation of the rabbit macrophages in the dermis by injection of Mycobacterium tuberculosis (BCG strain) at that site would be more protective than the previously used technique of intravenous injection of BCG (Graves and Johnson, 1975), resulting mainly in activated macrophages in liver and spleen. This is because the $T$. pallidum challenge is normally given intradermally into the shaved back of the rabbit. Lukehart and Miller (1978) have previously commented on the need to activate macrophages at the actual site of $T$. pallidum challenge before drawing conclusions about their function in this disease. They have demonstrated macrophage uptake of $T$. pallidum in vitro.

As reported in this paper, rabbits stimulated with BCG were still infected with $T$. pallidum and no protection was evident, suggesting that activated macrophages do not play a significant part in resistance to reinfection in rabbit syphilis. Baughn et al. (1977a) came to a similar conclusion after intradermally injected Propionibacterium acnes failed to protect rabbits against an intradermal challenge with $T$. pallidum.

\section{Materials and methods}

All rabbit groups (A, B, C, D, and E) contained three rabbits (Table). 
Table Inoculation of five groups of rabbits with Mycobacterium bovis (BCG) and Treponema pallidum

\begin{tabular}{|c|c|c|c|c|}
\hline \multirow[b]{3}{*}{$\begin{array}{l}\text { Experimental } \\
\text { groups }\end{array}$} & \multicolumn{4}{|c|}{ Inoculation time } \\
\hline & \multirow{2}{*}{$\begin{array}{l}\text { Zero } \\
B C G \\
(2 \mathrm{mg} \text { i.v. })\end{array}$} & \multicolumn{2}{|c|}{ One month* } & \multirow{2}{*}{$\frac{\text { Six months }}{\text { T. pallidum } \S}$} \\
\hline & & $B C G+$ & T. pallidum $\neq$ & \\
\hline $\begin{array}{l}\text { A } \\
\text { B } \\
\text { C } \\
\text { D } \\
\text { E }\end{array}$ & $\begin{array}{l}\text { Yes } \\
\text { Yes } \\
\text { No } \\
\text { No } \\
\text { No }\end{array}$ & $\begin{array}{l}\text { Yes } \\
\text { No } \\
\text { Yes } \\
\text { No } \\
\text { No }\end{array}$ & $\begin{array}{l}\text { Yes } \\
\text { Yes } \\
\text { Yes } \\
\text { Yes } \\
\text { No }\end{array}$ & $\begin{array}{l}\text { Yes } \\
\text { Yes } \\
\text { Yes } \\
\text { Yes } \\
\text { Yes }\end{array}$ \\
\hline
\end{tabular}

${ }^{*}$ In groups $\mathrm{A}$ and $\mathrm{C}$ the $\mathrm{BCG}$ and $T$. pallidum challenge was given simultaneously in the same syringe after being mixed within five minutes of inoculation.

†Eight sites were inoculated; $250 \mu \mathrm{g} / \mathrm{site}$, total $2 \mathrm{mg}$.

Fight sites were inoculated; two each with $10^{4}, 10^{3}, 10^{2}$, and 10 intradermally.

$\S$ Eight sites were inoculated; two each with $10^{6}, 10^{5}, 10^{4}$, and $10^{3}$ intradermally.

\section{INFECTION WITH BCG}

\section{At zero time}

Rabbits in groups A and B (Table) were pretreated with $2 \mathrm{mg} \mathrm{BCG}$ (dry weight) given intravenously in saline one month before the first challenge with $T$. pallidum. BCG was obtained from the Commonwealth Serum Laboratories, Melbourne, Australia.

\section{At one month}

Rabbits in groups A and C (Table) were infected with BCG at the same time as the first challenge with $T$. pallidum (see below). The BCG was inoculated into eight intradermal sites on the shaved back of each rabbit; $250 \mu \mathrm{g} \mathrm{BCG} \mathrm{(dry} \mathrm{weight)} \mathrm{was} \mathrm{inoculated} \mathrm{per}$ site, a total of $2 \mathrm{mg}$ per rabbit.

\section{CHALLENGE WITH $T$. PALLIDUM At one month}

All rabbit groups, except $\mathrm{E}$ (Table), were challenged at eight intradermal shaved sites per rabbit with duplicate doses of $10^{4}, 10^{3}, 10^{2}$, and $10 T$. pallidum (see below for preparation). The BCG and $T$. pallidum were independently suspended in anaerobic bacterial medium (Graves et al., 1975) and mixed together immediately before inoculation into the rabbit using the same syringe.

\section{At six months}

All rabbit groups (Table) were challenged at eight intradermal shaved sites per rabbit with duplicate doses of $10^{6}, 10^{5}, 10^{4}$, and $10^{3} \mathrm{~T}$. pallidum.

\section{MONITORING THE COURSE OF INFECTION}

After the first challenge with $T$. pallidum rabbits were examined daily for one month, every two or three days thereafter for another month, and every week thereafter for a further $21 / 2$ months, a total observation period of $4 \frac{1}{2}$ months. After the challenge with $T$. pallidum at six months, rabbits were observed for a total of 2 months, although no further lesions appeared after the first $3 \frac{1}{2}$ weeks. The rabbits were shaved regularly and the degree of induration was recorded. The appearance of any secondary syphilitic lesions was noted. The experimental rabbits were individually housed at $16-19^{\circ} \mathrm{C}$ and fed antibiotic-free rabbit pellets and water in unrestricted quantities. Only male rabbits were used.

\section{PREPARATION OF CHALLENGE T. PALLIDUM}

$T$. pallidum was propagated in the testes of male rabbits by inoculating $5 \times 10^{7}$ viable $T$. pallidum per testis and harvesting it approximately 11 days later when a well developed orchitis was present. Elution of the $T$. pallidum from the minced orchitic testis required several sequential five-minute washings with $5-10 \mathrm{ml}$ of sterile, prereduced anaerobic medium (Graves et al., 1975). The washings were pooled under a flow of sterile oxygen-free nitrogen, counts were made in a Petroff-Hauser chamber, and the suspension was diluted to the required concentration for rabbit challenge.

SEROLOGICAL TESTING OF INFECTED RABBITS To test for anti-cardiolipin (reaginic) antibody the rapid plasma reagin (RPR) card test (Hynson, Wescott and Dunning, Baltimore) was used qualitatively. To test for anti-treponemal antibody the $T$. pallidum haemagglutination (TPHA) test (Fujizoki Pharmaceutical Co. Ltd., Shinjuku-ku, Tokyo) was used quantitatively. In the latter test titres of $1 / 80$ or greater were considered to indicate antigenic stimulation with $T$. pallidum.

\section{Results}

\section{SINGLE INFECTION WITH BCG (GROUPS B AND C) \\ At zero time}

When rabbits in group B were challenged with $T$. pallidum (at one month) dermal syphilitic lesions appeared after incubation periods which were almost identical to those of control rabbits (group D). At the $10^{4}$ challenge sites lesions appeared after 14 days (control 13 days); at the $10^{3}$ challenge sites after 15 days (control 17 days); at the $10^{2}$ challenge sites after 17 days (control 18 days); and at the 10 challenge site after 19 days (control 18 days). These incubation periods represent the mean of six lesions (two per rabbit for each of three rabbits). Thus BCG infection at zero time did not modify the incubation period of the subsequent syphilitic infection indicating that it 
did not retard the growth of $T$. pallidum in these rabbits.

\section{At one month}

Rabbits in group C received their BCG infection at the same time and same site as their first challenge with $T$. pallidum. They did not receive BCG infection at zero time. Three days after infection they developed indurations of approximately $1 \mathrm{~cm}$ diameter at the sites of infection. These lesions faded after three to four weeks and disappeared by five weeks. All eight inoculation sites per rabbit showed similar indurated lesions, which appeared and disappeared together, unlike the control rabbits (group D) in which the sequential appearance of syphilitic lesions paralleled the size of the $T$. pallidum inoculum at that site. For this reason the lesions seemed to be due primarily to the growth of BCG, since each inoculation site received the same dose of BCG $(250 \mu \mathrm{g})$. In two of the three rabbits in group C, the indurated lesions, after disappearing, reappeared again in a milder form at the original sites of inoculation about two months after the initial infection. They persisted for another two to four weeks before again disappearing. This further suggested that the indurations were due to BCG infection because $T$. pallidurn lesions never reappear at the same site once they have regressed. Apparently the BCG growth masked any $T$. pallidum lesion development. The $T$. pallidum was not destroyed, however, since the rabbits subsequently developed syphilitic immunity. Furthermore, two of the three rabbits in group C did develop secondary, dermal, syphilitic lesions at sites on the shaved backs of the rabbits other than those inoculated. These appeared between day 36 and day 40 after inoculation, which is the normal time for secondary lesions to appear in $T$. pallidum-infected (but otherwise untreated) rabbits that do develop secondary lesions. The appearance of secondary lesions usually occurs in only a few rabbits after intradermal inoculation (Chesney and Schipper, 1950; Turner and Hollander, 1957).

DOUBLE INFECTION WITH BCG (GROUP A)

Rabbits in group $A$ received two infections with BCG, one at zero time and another one month later. One to two days after the BCG infection at one month (and associated $T$. pallidum challenge) all eight inoculation sites showed strong delayed type hypersensitivity (DHS) reactions of equal intensity. However the induration did not fade, as would be the case with normal DHS, but remained strong (greater than $1 \mathrm{~cm}$ diameter) until about one month after infection. No sign of the classical syphilitic lesion (neither primary nor secondary) appeared on these rabbits, and, as before, the lesion seemed to be due primarily to BCG growth or host hypersensitivity to BCG or both. Thus, $T$. pallidum growth was masked but evidently did occur because the rabbits subsequently developed syphilitic immunity.

CHALLENGE WITH T. PALLIDUM AT SIX MONTHS After a second infection with $T$. pallidum at six months, all the rabbits of group A, B, C, and D failed to develop any syphilitic lesions whereas the control rabbits of group $\mathrm{E}$ all developed lesions with the following average incubation times: five days $\left(10^{6}\right.$ inoculum); eight days ( $10^{5}$ inoculum); 14 days $\left(10^{4}\right.$ inoculum), and 21 days ( $10^{3}$ inoculum). This demonstrates that the initial $T$. pallidum inoculation in all groups, including groups $\mathbf{A}$ and $\mathbf{C}$ where no primary syphilitic lesions were observed, did result in infection as shown by the subsequent development of immunity to repeat challenge.

\section{SEROLOGICAL RESPONSES}

Anti-cardiolipin antibody was synthesised by all rabbits in groups $A, B, C$, and D between one and two months after infection. Anti-treponemal antibodies (TPHA test) were also synthesised by all the above rabbits by two months after infection with $T$. pallidum. Titres did not vary significantly from one group to another.

\section{Discussion}

Mycobacterial species, especially BCG, and chemical fractions derived from mycobacteria have been widely used to enhance non-specific cell-mediated immunity to a variety of micro-organisms and tumours. In the case of bacteria it acts by antigeninduced blast transformation of specific T-lymphocytes and the release of lymphokines which activate normal host macrophages to develop enhanced intracellular killing ability. This latter ability is mainly non-specific (Mackaness, 1971).

Earlier work by Graves and Johnson (1975) demonstrated that BCG given intravenously one month before intradermal challenge with $T$. pallidum failed to modify the development of syphilitic lesions in the rabbit. The simultaneous administration of immune syphilitic serum markedly delayed the onset of lesions but there was no synergy evident between the immune serum and the BCG, suggesting that opsonisation had not occurred. Schell et al. (1975b) also reported that BCG failed to suppress a challenge with intravenous $T$. pallidum in rabbits while Baughn et al. (1977a) noted that the use of Propionibacterium acnes as a macrophage stimulant failed to modify the course of an infection with $T$. pallidum in rabbits even when given intradermally. Harris and 
Thoen (1977) reported that the intradermal injection of BCG in mineral oil did protect rabbits from challenge with $T$. pallidum five days later. This finding is not consistent with the present observations, although differences in experimental procedure did occur.

There is evidence that cell-mediated immunity does develop in the host (rabbit or man) during the course of an infection with T. pallidum (Musher et al., 1976; Kiraly, 1976), and possibly it plays some role in the regression of lesions and the ultimate recovery of the host-for example, in human infections where recovery is sometimes spontaneous. Is phagocytosis by macrophages an important effector mechanism of cell-mediated immunity in syphilis? After in-vitro studies, Lukehart and Miller (1978) claim that it is and that immune serum significantly enhanced invitro phagocytosis of $T$. pallidum by rabbit peritoneal macrophages. If macrophages are important, why does BCG not enhance $T$. pallidum killing? Perhaps the activation of $T$. pallidum-killing macrophages is highly specific, requiring certain obligatory treponemal antigens, and cannot therefore be induced with BCG.

Alternatively, the macrophage may not play an important role in immunity. $T$. pallidum could be killed by some other mechanism, such as immune cytolysis involving specific T-lymphocytes or antibody-mediated K-cell killing. Electron microscopical observations of early syphilitic lesions have shown that very few $T$. pallidum are intracellular and that most are extracellular (Azar et al., 1970; Lauderdale and Goldman, 1972). This, of course, does not rule out a role for phagocytic cells later in the infection (during the immune stage) but it does suggest that rapid and efficient uptake of $T$. pallidum by normal host cells does not occur as part of the initial response to infection. Assuming that the $T$. pallidum cell is not susceptible to phagocytosis in the normal host, it is perhaps not surprising that enhancing the intracellular killing power of the macrophages with BCG does not result in enhanced killing of T. pallidum. Wicker et al. (1977) reported that leucocytes (monocytes and polymorphonuclear leucocytes) from syphilitic rabbits showed an increased incidence of nitroblue tetrazolium (NBT) reduction. This increase was observed 10 days after infection and reached a maximum 30 days after infection with $T$. pallidum; the NBT-positive leucocytes returned to normal 50 days after infection. This corresponds to the period taken for the lesion to heal but not to the period of maximum immunity to reinfection. Rabbits are not usually fully resistant to $T$. pallidum challenge until about three months after infection (Turner and Hollander, 1957). Does this suggest that phagocytosis and induction of immunity are temporally well separated? It is the immunologically more important macrophages that are the key to this question and not the polymorphonuclear leucocytes. Although human monocytes did not phagocytose virulent $T$. pallidum in vitro (Brause and Roberts, 1978), the role of the macrophage in the syphilitic lesion is still to be determined.

The inability of spleen cells from immune syphilitic rabbits to confer immunity to challenge (Baughn et al., 1977b), even with inbred recipient rabbits, implies that lymphocytes (of either subclass T or B) are not effector cells in the immune animal. Antibodies are the best documented line of defence against $T$. pallidum in rabbits, possibly acting in concert with some cellular elements of the host, since alone they do ameliorate infections but without giving complete protection. Metzger and Smogor (1975) reported some degree of immunity following passive transfer of lymphocytes from immune rabbit lymphnodes, but this would have included B (antibody-producing) lymphocytes, so the effect observed may have been due to antibody synthesis in the recipient rabbit.

The present study has shown the inability of BCG, a commonly used reticuloendothelial system stimulant, to stimulate the rabbit's immune system against $T$. pallidum. The injection of BCG into the same intradermal sites as the $T$. pallidum did mask the development of syphilitic lesions due to the growth of, or hypersensitivity to, BCG or both. The normal method of determining if these rabbits were infected with $T$. pallidum, in the absence of observable, characteristic lesions, is to wait until several months after inoculation and then to transfer excised lymph nodes to the testes of a normal rabbit. This latter rabbit is then examined regularly for a syphilitic orchitis in which $T$. pallidum is demonstrable by darkfield microscopy (Miller, 1971). This method was not used in this study owing to a shortage of space in our laboratory and because an equally sound technique was available that did not require additional rabbits. This method is based on the observation that after an untreated syphilitic infection of about three months' duration these rabbits are refractory to challenge with the same strain of $T$. pallidum. Hence, when no lesions are observed a rabbit can be tested for the presence of infection by challenging with $T$. pallidum at least three months after the time of suspected infection. In this experiment a time period of five months was used to be certain that immunity had developed in the infected rabbits. If, on challenge, no lesions develop the rabbits can be assumed to have been previously infected, thus explaining their current state of immunity. If, on the other hand, lesions did occur on challenge the implication is that the 
previous exposure to $T$. pallidum had not resulted in a syphilitic infection.

In the present study, all rabbits were shown to have been infected by the challenge with $T$. pallidum at one month by virtue of their immunity to the challenge with $T$. pallidum at six months. In support of this conclusion, all rabbits developed antibodies to cardiolipin (reagins) and $T$. pallidum antigens as shown by the RPR and the TPHA tests respectively. Although a serological response, as measured by the TPHA test, could have developed by simple antigenic stimulation and without an infection the development of cardiolipin antibodies represents tissue destruction caused by $T$. pallidum infection (Fredericksson et al., 1968).

The author wishes to thank Ian McLean for excellent technical assistance. The work was supported by grants from the National Health and Medical Research Council (Australia), the Utah Foundation, the Ian Potter Foundation, the Danks Trust, Monash University and the estate of the late George Adams, from whom funds are gratefully acknowledged.

\section{References}

Azar, H., Pham, T., and Kurbun, A. (1970). An electron microscopic study of a syphilitic chancre. Archives of Pathology, 90, 143-150.

Baughn, R., Musher, D., and Knox, J. (1977a). Effect of sensitization with Propionibacterium acnes on the growth of Listeria monocytogenes and Treponema pallidum in rabbits. Journal of Immunology, 118, 109-113.

Baughn, R., Musher, D., and Simmons, C. (1977b). Inability of spleen cells from chancre-immune rabbits to confer immunity to challenge with Treponema pallidum. Infection and Immunity, 17, 535-540.

Bishop, N. and Miller, J. (1976). Humoral immunity in experimental syphilis. I. The demonstration of resistance conferred by passive immunization. Journal of Immunology, 117, 191-196.

Brause, B. and Roberts, R. (1978). Attachment of virulent Treponema pallidum to human mononuclear phagocytes. British Journal of Venereal Diseases, 54, 218-224.

Cheaney, A. and Schipper, G. (1950). The effect of the method of inoculation on the course of experimental syphilis in the rabbit. American Journal of Syphilology, 34, 18-24.

Fredericksson, T., Hederstedt, B., and Rosengren, S. (1968). A study on the Wassermann and TPI antibodies in relation to histopathological findings in $T$. pallidum infected animals and man. Acta Pathologica et Microbiologica Scandinavica, 72, 125-138.

Graves, S. and Johnson, R. (1975). Effect of pretreatment with Mycobacterium bovis (strain BCG) and immune syphilitic serum on rabbit resistance to Treponema pallidum. Infection and Immunity, 12, 1029-1036.
Graves, S., Sandok, P., Jenkin, H., and Johnson, R. (1975). Retention of motility and virulence of Treponema pallidum (Nichols strain) in vitro. Infection and Immunity, 12, 1116-1120.

Harris, D. and Thoen, C. (1977). Inhibition of skin lesions due to Treponema pallidum in BCG-infected rabbits. Abstracts of Annual Meeting of American Society of Microbiology E32.

Kiraly, K. (1976). Immunoallergologic aspects of syphilis. In Immunological Aspects of Allergy and Allergic Diseases. Edited by E. Rajka and S. Korossy, Volume 8, Chapter 73, pp. 71-105. Plenum Press: New York.

Lauderdale, V. and Goldman, J. (1972). Serial ultrathin sectioning demonstrating the intracellularity of $T$. pallidum. British Journal of Venereal Diseases, 48, 87-96.

Levene, G., Wright, D., and Turk, J. (1971). Cell-mediated immunity and lymphocyte transformation in syphilis. Proceedings of the Royal Society of Medicine, 64, 426-428.

Lukehart, S. and Miller, J. (1978). Demonstration of the in vitro phagocytosis of Treponema pallidum by rabbit peritoneal macrophages. Journal of Immunology, 121, 2014-2024.

Mackaness, G. (1971). Resistance to intracellular infection. Journa of Infectious Diseases, 123, 439-445.

Metzger, K., Podwinska, J., and Smogor, W. (1977). Cell-mediated immunity in experimental syphilis in rabbits. Archives of Immunology and Experimental Therapy, 25, 25-34.

Metzger, M. and Smogor, W. (1975). Passive transfer of immunity to experimental syphilis in rabbits by immune lymphocytes Archives of Immunology and Experimental Therapy, 23, 625-630.

Miller, J. (Ed.) (1971). Spirochetes in body fluids and tissues. In Manual of Investigative Methods. Charles C. Thomas: Springfield, Illinois, USA

Musher, D., Schell, R., and Knox, J. (1976). The immunology of syphilis. International Journal of Dermatology, 15, 566-576.

Perine, P., Weiser, R., and Klebanoff, S. (1973). Immunity to syphilis. I. Passive transfer in rabbits with hyperimmune serum. Infection and Immunity, 8, 787-790.

Schell, R. and Musher, D. (1975). Transfer of non-specific resistance to Listeria monocytogenes using spleen cells from syphilitic rabbits. Proceedings of the Society of Experimental Biology and Medicine, 148, 516-518.

Schell, R., Musher, D., Jacobson, K., and Schwethelm, P. (1975a) Induction of acquired cellular resistance following transfer of thymus-dependent lymphocytes from syphilitic rabbits. Journal of Immunology, 114, 550-553.

Schell, R., Musher, D., Jacobson, K., Schwethelm, P., and Simmons, C. (1975b). Effect of macrophage activation on infection with Treponema pallidum. Infection and Immunity, 12 , 505-511.

Sepetjian, M., Salussola, D., and Thivolet, J. (1973). Attempt to protect rabbits against experimental syphilis by passive immunization. British Journal of Venereal Diseases, 49, 335-337.

Turner, T., Hardy, P., Newman, B., and Nell, E. (1973). Effects of passive immunization on experimental syphilis in the rabbit. Johns Hopkins Medical Journal, 133, 241-251.

Turner, T. and Hollander, D. (1957). Biology of the treponematoses. Monograph Series, No. 35. World Health Organisation: Geneva.

Weiser, R., Erickson, D., Perine, P., and Pearsall, N. (19:6). Immunity to syphilis: passive transfer in rabbits using serial doses of immune serum. Infection and Immunity, 13, 1402-1407.

Wicker, V., Blakowski, S., and Wicker, K. (1977). Nitroblue tetrazolium tests in experimental syphilis. British Journal of Venereal Diseases, 53, 292-294.

Wicker, V. and Wicker, K. (1975). Cell response in rabbits infected with $T$. pallidum as measured by the leucocyte migration inhibition test. British Journal of Venereal Diseases, 51, 240-245.

Wicker, V. and Wicker, K. (1977). In vitro cell response of Treponema pallidum infected rabbits. I. Lymphocyte transformation. Clinical and Experimental Immunology, 29, 480-486. 